\title{
Adaptive Control of Flexible Structures using Residual Mode Filters
}

\author{
Mark J. Balas, Fellow, IEEE and Susan A. Frost, Member, IEEE
}

\begin{abstract}
Flexible structures containing a large number of modes can benefit from adaptive control techniques which are well suited to applications that have unknown modeling parameters and poorly known operating conditions. In this paper, we focus on a direct adaptive control approach that has been extended to handle adaptive rejection of persistent disturbances. We extend our adaptive control theory to accommodate troublesome modal subsystems of a plant that might inhibit the adaptive controller.

In some cases the plant does not satisfy the requirements of Almost Strict Positive Realness. Instead, there maybe be a modal subsystem that inhibits this property. This section will present new results for our adaptive control theory. We will modify the adaptive controller with a Residual Mode Filter (RMF) to compensate for the troublesome modal subsystem, or the $Q$ modes. Here we present the theory for adaptive controllers modified by RMFs, with attention to the issue of disturbances propagating through the $Q$ modes. We apply the theoretical results to a flexible structure example to illustrate the behavior with and without the residual mode filter.
\end{abstract}

\section{INTRODUCTION}

$\mathrm{F}$ lexible structures containing a large number of modes can benefit from adaptive control techniques which are well suited to applications that have unknown modeling parameters and poorly known operating conditions. Creating an accurate model of the dynamic characteristics of a structure can be extremely difficult, if not impossible. In this paper, we focus on the direct adaptive control (DAC) approach developed in [1-2]. This approach has been extended to handle adaptive rejection of persistent disturbances [3] and applied to wind turbines in [4].

In this paper, we extend our adaptive control theory to accommodate modal subsystems of a plant that inhibit the adaptive controller, in particular those residual modes that interfere with the almost strict positive real condition.

A flexible structure Evolving System is a mechanical dynamical system consisting of actively controlled flexible structure components that are joined together by compliant forces. A practical and well-accepted representation of flexible structures is based on the finite element method (FEM); see [9] for an extensive survey on flexible structures. The FEM of the lumped model in physical coordinates $q$, for a linearized actively controlled flexible structure with $\mathrm{M}$ control inputs, and $\mathrm{P}$ control outputs is given in matrix form as

M. J. Balas is the Department Head of the Department of Electrical and Computer Engineering, University of Wyoming, Laramie, WY 82071 USA (mbalas@uwyo.edu).

S. A. Frost is in the Intelligent Systems Division, NASA Ames Research Center, Moffett Field, CA 94035 USA (susan.frost@nasa.gov).
$\left\{\begin{array}{l}M_{0} \ddot{q}+D_{0} \dot{q}+K_{0} q=B_{0} u_{p} \\ y_{p}=C_{0} q+E_{0} \dot{q}\end{array}\right.$

This system can be put into a modal form with the transformation

$q=\Phi_{0} \eta$

where $\left\{\begin{array}{l}\Phi_{0}^{T} M_{0} \Phi_{0}=I \\ \Phi_{0}^{T} K_{0} \Phi_{0}=\Lambda_{0} \equiv \operatorname{diag}\left[\omega_{k}^{2}\right]\end{array}\right.$

Therefore, using the transformation (2), we obtain the modal form of (1):

$\left\{\begin{array}{l}\ddot{\eta}+\bar{D}_{0} \dot{\eta}+\bar{\Lambda}_{0} \eta=\bar{B}_{0} u_{p} \\ y_{p}=\bar{C}_{0} \eta+\bar{E}_{0} \dot{\eta}\end{array}\right.$

This system can be put into a modal first-order form with the states $x_{p}^{T} \equiv\left[\begin{array}{ll}\eta & \dot{\eta}\end{array}\right]$.

Note that many kinds of systems have modal forms, and the results we are developing here apply to any such system, not just flexible structures.

\section{Direct AdAPtive CONTROL With REJECTION OF PERSISTENT DisTURBANCES}

We give relevant details of this theory here. The plant is assumed to be well modeled by the linear, time-invariant, finite-dimensional system:

$\left\{\begin{array}{l}\dot{x}_{p}=A_{p} x_{p}+B_{p} u_{p}+\Gamma_{p} u_{D} \\ y_{p}=C_{p} x_{p} ; x_{p}(0)=x_{0}\end{array}\right.$

where the plant state, $x_{p}$ is an $\mathrm{N}_{\mathrm{p}}$-dimensional vector, the control input vector, $u_{p}$, is $\mathrm{M}$-dimensional, and the sensor output vector, $y_{p}$, is $\mathrm{P}$-dimensional. The disturbance input vector, $u_{D}$, is $\mathrm{M}_{\mathrm{D}}$-dimensional and will be thought to come from the Disturbance Generator:

$$
\left\{\begin{array}{l}
u_{D}=\Theta z_{D} \\
\dot{z}_{D}=F z_{D} ; z_{D}(0)=z_{0}
\end{array}\right.
$$

where the disturbance state, $z_{D}$, is $\mathrm{N}_{\mathrm{D}}$-dimensional. All matrices in (4)-(5) have the appropriate compatible dimensions. Such descriptions of persistent disturbances were first used in [5] to describe signals of known form but unknown amplitude. Equation (5) can be rewritten in a form that is not a dynamical system, which is sometimes easier to use:

$\left\{\begin{array}{l}u_{D}=\theta z_{D} \\ z_{D}=L \phi_{D}\end{array}\right.$

where $\phi_{D}$ is a vector composed of the known basis 
functions for the solution of $u_{D}=\Theta z_{D}$, i.e., $\phi_{D}$ are the basis functions which make up the known form of the disturbance, and $L$ is a matrix of dimension $\mathrm{N}_{\mathrm{D}}$ by $\operatorname{dim}\left(\phi_{D}\right)$. The method for rejecting persistent disturbances used in this paper requires only the knowledge of the form of the disturbance, the amplitude of the disturbance does not need to be known, i.e. $(L, \Theta)$ can be unknown.

In much of the control literature, it is assumed that the plant and disturbance generator parameter matrices $(A, B, C, \Gamma, \Theta, F)$ are known. This knowledge of the plant and its disturbance generator allows the Separation Principle of Linear Control Theory to be invoked to arrive at a StateEstimator based, linear controller which can suppress the persistent disturbances via feedback. In this paper, we will not assume that the plant and disturbance generator parameter matrices $(A, B, C, \Gamma, \Theta)$ are known. But, we will assume that we know the disturbance generator parameter, $F$, from (5), i.e., the form of the disturbance functions is known. In many cases, knowledge of $F$ is not a severe restriction, since the disturbance function is often of known form but unknown amplitude.

Our control objective will be to cause the output of the plant, $y_{p}$, to asymptotically track zero while accommodating disturbances of the form given by the disturbance generator. We define the output error vector as:

$e_{y} \equiv y_{p}-0$

To achieve the desired control objective, we want

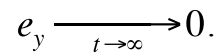

Consider the plant given by (4) with the disturbance generator given by (6). The control objective for this system will be accomplished by an adaptive control law of the form:

$u_{p}=G_{e} e_{y}+G_{D} \phi_{D}$

where $G_{e}$ and $G_{D}$ are matrices of the appropriate compatible dimensions, whose definitions will be given later. In [8], the gain adaptation laws were developed to make asymptotic output regulation possible.

Now we specify the adaptive gain laws, which produce asymptotic tracking:

$\left\{\begin{array}{l}\dot{G}_{e}=-e_{y} e_{y}^{T} h_{11} \\ \dot{G}_{D}=-e_{y} \phi_{D}^{T} h_{22}\end{array}\right.$

where $h_{11}$ and $h_{22}$ are arbitrary, positive definite matrices. The adaptive controller is specified by (9) with the above adaptive gain laws (10). See [3] for the stability analysis of this controller and proof that the adaptive gains, $G_{e}$ and $G_{D}$, remain bounded and asymptotic tracking occurs, i.e., $e_{y} \underset{t \rightarrow \infty}{\longrightarrow} 0$.

\section{Residual Mode Filter Augmentation of AdAPtive CONTROLlER}

In some cases the plant in (4) does not satisfy the requirements of ASPR. Instead, there may be a modal subsystem that inhibits this property. This section will present new results for our adaptive control theory. We will modify the adaptive controller with a Residual Mode Filter (RMF) to compensate for the troublesome modal subsystem, or the Q modes, as was done in [6] for fixed gain nonadaptive controllers. Here we present the theory for adaptive controllers modified by RMFs. In a previous paper, we examined the RMF with adaptive control, but assumed that there was no leakage of the disturbance into the Q modes [7]. Here we will deal with the issue of disturbances propagating through these modes.

Let us assume that (4) can be partitioned into the following modal form:

$\left\{\begin{array}{l}{\left[\begin{array}{c}\dot{x} \\ \dot{x}_{Q}\end{array}\right]=\left[\begin{array}{ll}A & 0 \\ 0 & A_{Q}\end{array}\right]\left[\begin{array}{c}x \\ x_{Q}\end{array}\right]+\left[\begin{array}{c}B \\ B_{Q}\end{array}\right] u_{p}+\left[\begin{array}{c}\Gamma \\ \varepsilon \Gamma_{Q}\end{array}\right] u_{D}} \\ y_{p}=\left[\begin{array}{ll}C & C_{Q}\end{array}\right]\left[\begin{array}{c}x \\ x_{Q}\end{array}\right] ; \varepsilon \geq 0\end{array}\right.$

Define $x_{p} \equiv\left[\begin{array}{c}x \\ x_{Q}\end{array}\right] ; A_{p}=\left[\begin{array}{cc}A & 0 \\ 0 & A_{Q}\end{array}\right] ; B_{p}=\left[\begin{array}{c}B \\ B_{Q}\end{array}\right]$;

$\Gamma_{p}=\left[\begin{array}{ll}\Gamma & \varepsilon \Gamma_{Q}\end{array}\right]^{T} ; C_{p}=\left[C C_{Q}\right]$ and Disturbance

Generator $\left\{\begin{array}{l}\dot{z}_{D}=F z_{D} \\ u_{D}=\theta z_{D}\end{array}\right.$ or $z_{D}=L \phi_{D}$ as before in (5)-(6).

The Output Tracking Error and control objective remain as in (7)-(8), i.e. $e_{y} \equiv y_{p} \longrightarrow t \rightarrow \infty$.

However, now we will only assume that the subsystem $(A, B, C)$ is Almost Strictly Positive Real (ASPR), rather than the full un-partitioned plant $\left(A_{p}, B_{p}, C_{p}\right)$, and the modal subsystem $\left(A_{Q}, B_{Q}, C_{Q}\right)$ will be known and openloop stable, i.e. $A_{Q}$ is stable. Also note that this subsystem is directly affected by the disturbance input. Recall that ASPR means $C B>0$ and $P(s)=C(s I-A)^{-1} B$ is minimum phase. So, in summary, the actual plant has an ASPR subsystem and a known modal subsystem that is stable but inhibits the property of ASPR for the full plant. Hence, this modal subsystem must be compensated or filtered away.

We define the Residual Mode Filter (RMF):

$\left\{\begin{array}{l}\dot{\hat{x}}_{Q}=A_{Q} \hat{x}_{Q}+B_{Q} u_{p} \\ \hat{y}_{Q}=C_{Q} \hat{x}_{Q}\end{array}\right.$

And the compensated tracking error:

$\tilde{e}_{y} \equiv y_{p}-\hat{y}_{Q}$

Now we let $e_{Q} \equiv \hat{x}_{Q}-x_{Q}$ and obtain:

$\dot{e}_{Q}=A_{Q} e_{Q}-\varepsilon \Gamma_{Q} u_{D}$

Consequently,

$\begin{aligned} \tilde{e}_{y} & \equiv y_{p}-\hat{y}_{Q}=C x+C_{Q} x_{Q}-\left[C_{Q} x_{Q}+C_{Q} e_{Q}\right] \\ & =C x-C_{Q} e_{Q}\end{aligned}$

As in [1]-[2], we define the Ideal Trajectories, but only for 
the ASPR Subsystem:

$\left\{\begin{array}{l}\dot{x}_{*}=A x_{*}+B u_{*}+\Gamma u_{D} \\ y_{*}=C x_{*}=0\end{array}\right.$

where $\left\{\begin{array}{l}x_{*} \equiv S_{1}^{*} z_{D} \\ u_{*} \equiv S_{2}^{*} z_{D}\end{array}\right.$

This is equivalent to the Matching Conditions:

$$
\left\{\begin{array}{l}
S_{1}^{*} F=A S_{1}^{*}+B S_{2}^{*}+\Gamma \theta \\
C S_{1}^{*}=0
\end{array}\right.
$$

which are known to be uniquely solvable when $C B$ is nonsingular. However, we do not need to know the actual solutions for our adaptive control approach.

$$
\begin{aligned}
& \text { Let }\left\{\begin{array}{l}
\Delta x \equiv x-x_{*} \\
\Delta u \equiv u_{p}-u_{*} \\
\Delta \tilde{y} \equiv \tilde{e}_{y}=C x-C_{Q} e_{Q}
\end{array}\right. \text {. Then we have } \\
& \left\{\begin{array}{l}
\Delta \dot{x}=A \Delta x+B \Delta u \\
\Delta \tilde{y}=C x-y_{*}-C_{Q} e_{Q}=C \Delta x-C_{Q} e_{Q}
\end{array}\right.
\end{aligned}
$$

because, from (16), $y_{*}=0$. This system can be rewritten:

$$
\left\{\begin{aligned}
{\left[\begin{array}{c}
\Delta \dot{x} \\
\dot{e}_{Q}
\end{array}\right] } & =\left[\begin{array}{cc}
A & 0 \\
0 & A_{Q}
\end{array}\right]\left[\begin{array}{l}
\Delta x \\
e_{Q}
\end{array}\right]+\left[\begin{array}{c}
B \\
0
\end{array}\right] \Delta u+\left[\begin{array}{c}
0 \\
\varepsilon \Gamma_{Q}
\end{array}\right] u_{D} \\
& =\bar{A}\left[\begin{array}{l}
\Delta x \\
e_{Q}
\end{array}\right]+\bar{B} \Delta u+\varepsilon \bar{\Gamma}_{Q} u_{D} \\
\Delta \tilde{y} & =\left[\begin{array}{ll}
C & -C_{Q}
\end{array}\right]\left[\begin{array}{l}
\Delta x \\
e_{Q}
\end{array}\right]=\bar{C}\left[\begin{array}{l}
\Delta x \\
e_{Q}
\end{array}\right]
\end{aligned}\right.
$$

Now we have the following:

Lemma: $\left(\bar{A}=\left[\begin{array}{cc}A & 0 \\ 0 & A_{Q}\end{array}\right], \bar{B}=\left[\begin{array}{l}B \\ 0\end{array}\right], \bar{C}=\left[\begin{array}{ll}C & -C_{Q}\end{array}\right]\right)$ ASPR if and only if $(A, B, C)$ ASPR.

Proof: $\bar{C} \bar{B}=\left[\begin{array}{ll}C & -C_{Q}\end{array}\right]\left[\begin{array}{l}B \\ 0\end{array}\right]=C B>0$ and

$$
\begin{aligned}
\bar{P}(s) & \equiv \bar{C}(s I-\bar{A})^{-1} \bar{B} \\
& =\left[\begin{array}{ll}
C & -C_{Q}
\end{array}\right]\left[\begin{array}{cc}
(s I-A)^{-1} & 0 \\
0 & \left(s I-A_{Q}\right)^{-1}
\end{array}\right]\left[\begin{array}{l}
B \\
0
\end{array}\right] \\
& =C(s I-A)^{-1} B=P(s)
\end{aligned}
$$

is minimum phase and the result is proved \#

From this Lemma, there exists $G_{e}^{*}$ such that

$\left(\bar{A}_{C} \equiv \bar{A}+\bar{B} G_{e}^{*} \bar{C}, \bar{B}, \bar{C}\right)$ is Strictly Positive Real (SPR) when $(A, B, C)$ is ASPR. Consequently, as is well known from the Kalman-Yacubovic Theorem:

$\exists \bar{P}, \bar{Q}>0 \ni\left\{\begin{array}{l}\bar{A}_{C}^{T} \bar{P}+\bar{P} \bar{A}_{C}=-\bar{Q} \\ \bar{P} \bar{B}=\bar{C}^{T}\end{array}\right.$ $\left\{\begin{array}{l}u_{p} \equiv G_{e} \tilde{e}_{y}+G_{D} \phi_{D} \\ \tilde{e}_{y} \equiv y_{p}-\hat{y}_{Q} \\ \dot{\hat{x}}_{Q}=A_{Q} \hat{x}_{Q}+B_{Q} u_{p} \\ \hat{y}_{Q}=C_{Q} \hat{x}_{Q}\end{array}\right.$

with modified adaptive gains:

$\left\{\begin{array}{l}\dot{G}_{e}=-\tilde{e}_{y} \tilde{e}_{y}^{T} h_{e} ; h_{e}>0 \\ \dot{G}_{D}=-\tilde{e}_{y} \phi_{D}^{T} h_{D} ; h_{D}>0\end{array}\right.$

Finally, we have the following stability result:

Theorem: In (11), let $(A, B, C)$ ASPR, $A_{Q}$ stable, $\phi_{D}$ bounded. Then the Modified Adaptive Controller with RMF in (21)-(22) produces $e_{y}=y_{p}$ and $e_{Q}$ ultimately bounded into a ball of radius $R_{*} \equiv \varepsilon \frac{\left(1+\sqrt{p_{\max }}\right)}{a \sqrt{p_{\min }}} M_{v}$ with exponential rate and bounded adaptive gains $\left(G_{e}, G_{D}\right)$.

Proof: From (21),

$u_{p} \equiv G_{e} \tilde{e}_{y}+G_{D} \phi_{D} \Rightarrow$

$$
\begin{aligned}
& \Delta u \equiv u_{p}-u_{*} \\
& =\left[G_{e} \tilde{e}_{y}+G_{D} \phi_{D}\right]-\left[S_{2}^{*} L\right] \phi_{D} \\
& =G_{e}^{*} \tilde{e}_{y}+\Delta G \eta \\
& \text { where }\left\{\begin{array}{l}
\Delta G_{e} \equiv G_{e}-G_{e}^{*} \\
\Delta G_{D} \equiv G_{D}-\left(S_{2}^{*} L\right) \\
\Delta G \equiv G-G_{*}=\left[\begin{array}{ll}
\Delta G_{e} & \Delta G_{D}
\end{array}\right] \\
\eta \equiv\left[\begin{array}{l}
\tilde{e}_{y} \\
\phi_{D}
\end{array}\right]
\end{array}\right.
\end{aligned}
$$

Then

$\left\{\begin{array}{l}\dot{\zeta}=\bar{A} \zeta+\bar{B} \Delta u=\bar{A}_{C} \zeta+\bar{B} w+\varepsilon \bar{\Gamma}_{Q} u_{D} \\ \tilde{e}_{y}=\bar{C} \zeta\end{array}\right.$

with $\zeta \equiv\left[\begin{array}{l}\Delta x \\ e_{Q}\end{array}\right], w \equiv \Delta G \eta, \bar{A}_{C} \equiv \bar{A}+\bar{B} G_{e}^{*} \bar{C}$

From (22), we can see that

$$
\dot{G}=\Delta \dot{G}=-\tilde{e}_{y} \eta^{T} h ; h \equiv\left[\begin{array}{cc}
h_{e} & 0 \\
0 & h_{D}
\end{array}\right]>0
$$

Since $(A, B, C)$ is ASPR, and by the lemma, so is $(\bar{A}, \bar{B}, \bar{C})$, we can we can use the following result from [8] where $v \equiv \bar{\Gamma}_{Q} u_{D}$ is bounded because the disturbance $u_{D}=L \phi_{D}$ is bounded:

Result: Consider the nonlinear, coupled system of differential equations,

We now modify the adaptive control law with RMF: 


$$
\left\{\begin{array}{l}
\dot{\zeta}=\bar{A}_{c} \varsigma+\bar{B}\left(G(t)-G^{*}\right) \eta+\varepsilon v \\
\widetilde{e}_{y}=\bar{C} \varsigma \\
\dot{G}(t)=-\widetilde{e}_{y} \eta^{\mathrm{T}} h-a G(t)
\end{array}\right.
$$

where $G^{*}$ is any constant matrix and $h$ is any positive definite constant matrix, each of appropriate dimension. Assume the following:

i) the triple $(\bar{A}, \bar{B}, \bar{C})$ is $S P R$,

ii) $\exists M_{K}>0 \ni\left\|\left(G^{*}\right)^{T} G^{*}\right\| \leq M_{K}$, using the trace norm,

iii) $\exists M_{v}>0 \ni \sup _{t \geq 0}\|v(t)\| \leq M_{v}$,

iv) $\exists a>0 \ni a \leq \frac{q_{\min }}{2 p_{\max }}$, and

v) $h$ satisfies $\left\|h^{-1}\right\|_{2} \leq\left(\frac{\varepsilon M_{v}}{a M_{K}}\right)^{2}$, where $p_{\min }$, $p_{\max }$ are the minimum and maximum eigenvalues of $\bar{P}$ and $q_{\min }$ is the minimum eigenvalue of $\bar{Q}$ in $\left\{\begin{array}{l}\bar{A}_{C}^{T} \bar{P}+\bar{P} \bar{A}_{C}=-\bar{Q} \\ \bar{P} \bar{B}=\bar{C}^{T}\end{array}\right.$

Then the matrix $G(t)$ is bounded and the state $\zeta(t)$ exponentially approaches the ball of radius

$$
R_{*} \equiv \varepsilon \frac{\left(1+\sqrt{p_{\max }}\right)}{a \sqrt{p_{\min }}} M_{v} \text { with } \varepsilon>0 .
$$

From this result, we have $\zeta$ is ultimately bounded into the ball of radius $R_{*}$, which leads to both $e_{y} \equiv y_{p}=y_{p}-y_{*}=C \Delta x=\left[\begin{array}{ll}C & 0\end{array}\right] \zeta$ and $e_{Q}$ ultimately

bounded as well. Therefore $G=G_{*}+\Delta G$ is bounded, as desired. \#

Consequently, the radius of the error ball $R_{*} \equiv \varepsilon \frac{\left(1+\sqrt{p_{\max }}\right)}{a \sqrt{p_{\min }}} M_{v}$ is determined by the size of $\varepsilon$, which is related to the amount of disturbance leakage into the $\mathrm{Q}$ modes. It can be seen that, when there is no leakage of the disturbance into the Q modes $(\varepsilon=0)$, the convergence is asymptotic to zero.

\section{Simulation Results With RMF}

In this section we will apply the above theoretical results to a simple flexible structure example to illustrate the behavior with and without the residual mode filter. The structure has a rigid body mode and two flexible modes:

$$
\begin{aligned}
P(s) & =\frac{1+s}{s^{2}}-\frac{3}{s^{2}+s+1}+\frac{1}{s^{2}+s+2} \\
& =\frac{s^{5}+s^{4}+3 s^{3}+0 s^{2}+3 s+1}{s^{6}+2 s^{5}+4 s^{4}+3 s^{3}+2 s^{2}}
\end{aligned}
$$

This plant has non-minimum phase zeros at $0.422 \pm 0.9543 i$, and thus does not meet the ASPR condition.

However, when the middle mode $P_{Q}(s)=-\frac{s}{s^{2}+s+1}$ is removed, the plant becomes:

$P(s)=\frac{1+s}{s^{2}}+\frac{1}{s^{2}+s+2}=\frac{s^{3}+3 s^{2}+3 s+2}{s^{4}+s^{3}+2 s^{2}}$

which is minimum phase and has a state space realization:

$$
A=\left[\begin{array}{cccc}
0 & 1 & 0 & 0 \\
0 & 0 & 1 & 0 \\
0 & 0 & 0 & 1 \\
0 & 0 & -2 & -1
\end{array}\right], B=\left[\begin{array}{l}
0 \\
0 \\
0 \\
1
\end{array}\right], C=\left[\begin{array}{llll}
2 & 3 & 3 & 1
\end{array}\right]
$$

with $\mathrm{CB}=1>0$. Therefore, $(A, B, C)$ is ASPR.

The RMF generated by $P_{Q}(s)=-\frac{3}{s^{2}+s+1}$ is

$A_{Q}=\left[\begin{array}{cc}0 & 1 \\ -1 & -1\end{array}\right], B_{Q}=\left[\begin{array}{l}0 \\ 1\end{array}\right], C_{Q}=\left[\begin{array}{ll}-3 & 0\end{array}\right]$ and so $C_{Q} B_{Q}=0$.

The adaptive controller (21)-(22) is implemented with $h_{e} \equiv 10, h_{D} \equiv 100, a \equiv 50$. The disturbance is a step of size 10. Setting $\varepsilon=1$, we obtain Figures 1 and 2 from a MatLab/Simulink simulation. The output trace is shown to converge in fig. 1 with a bias of 4 . The adaptive gains also converge in fig. 2. This illustrates the behavior of the adaptive controller plus the second order RMF. Without the $R M F$, the plant and adaptive controller are unstable in closed-loop.

\section{CONCLUSION}

We have proposed a modified adaptive controller with a residual mode filter. The RMF is used to accommodate troublesome modes in the system that might otherwise inhibit the adaptive controller, in particular the ASPR condition. This new theory accounts for leakage of the disturbance term into the $\mathrm{Q}$ modes. A simple three-mode example shows that the RMF can restore stability to an otherwise unstable adaptively controlled system. This is done without modifying the adaptive controller design.

\section{REFERENCES}

[1] Wen, JT, Balas, MJ. Robust adaptive control in Hilbert space. Journal of Mathematical Analysis and Application 1989; 143(1): 1-26.

[2] Balas, MJ. Finite-dimensional direct adaptive control for discrete-time infinite-dimensional linear systems. Journal of Mathematical Analysis and Applications 1995; 196(1): 153-171.

[3] Fuentes, RJ, Balas, MJ. Direct adaptive rejection of persistent disturbances. Journal of Mathematical Analysis and Applications 2000; 251(1): 28-39.

[4] Frost, SA, Balas, MJ, and Wright, AD. Direct adaptive control of a utility-scale wind turbine for speed regulation, International Journal of Robust and Nonlinear Control, 2009, 19(1): 59-71, DOI: 10.1002/rnc.1329.

[5] Johnson, C.D. Theory of disturbance-accommodating controllers. Control \& Dynamic Systems, Advances in Theory and Applications, Leondes, CT. ed. Academic Press: New York, 1976; 12: 387-489.

[6] Balas, MJ, Finite-dimensional controllers for linear distributed parameter systems: Exponential stability using Residual Mode 
Filters," J. Mathematical Analysis \& Applications, Vol. 133, pp. 283296, 1988.

[7] Frost, S. A., Balas, M. J., and Wright, A. D. "Modified adaptive control for region 3 operation in the presence of wind turbine structural modes", Proceedings $49^{\text {th }}$ AIAA Aerospace Sciences Meeting, Orlando, 2010.

[8] Fuentes, R J and Balas, M J, "Robust Model Reference Adaptive Control with Disturbance Rejection", Proceedings of the American Control Conference, 2002.

[9] Balas, M., "Trends in Large Space Structure Control Theory: Fondest Hopes; Wildest Dreams," IEEE Trans. Automatic Control, AC-27, 522-535, 1982.

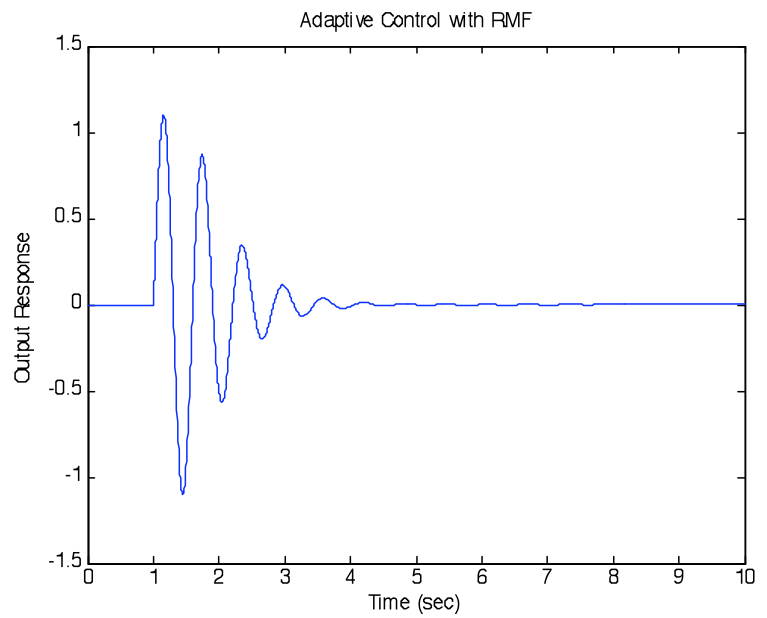

Fig. 1. Non-dimensional output response with adaptive controller augmented with RMF.

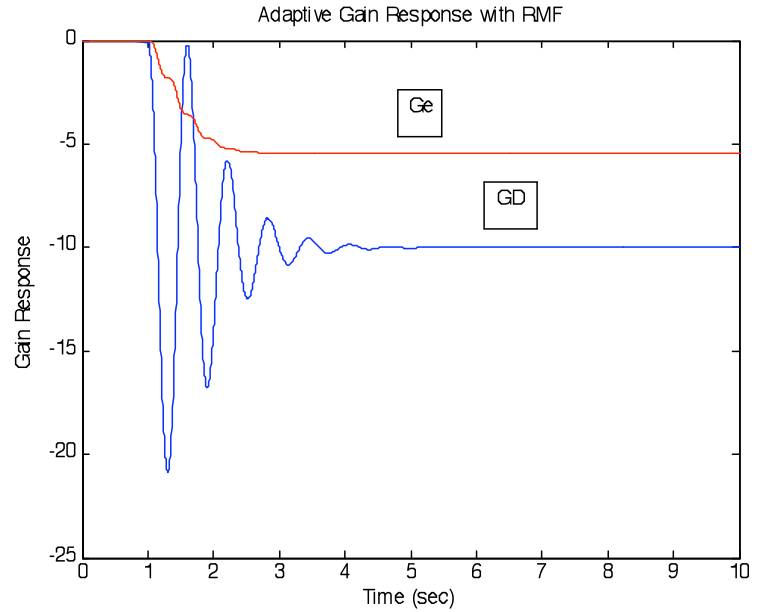

Fig. 2. Adaptive gains, $\mathrm{Ge}=$ error gain, $\mathrm{Gd}=$ disturbance gain. 